\title{
Design of a smart SiPM based on focal-plane processing elements for improved spatial resolution in PET
}

\author{
F. Pozas-Flores, R. Carmona-Galán, J. Fernández-Berni and Á. Rodríguez-Vázquez \\ Institute of Microelectronics of Seville (IMSE-CNM), CSIC-University of Seville, Spain.
}

\begin{abstract}
Single-photon avalanche diodes are compatible with standard CMOS. It means that photo-multipliers for scintillation detectors in nuclear medicine (i. e. PET, SPECT) can be built in inexpensive technologies. These silicon photo-multipliers consist in arrays of, usually passively-quenched, SPADs whose output current is sensed by some analog readout circuitry. In addition to the implementation of photosensors that are sensitive to singlephoton events, analog, digital and mixed-signal processing circuitry can be included in the same CMOS chip. For instance, the SPAD can be employed as an event detector, and with the help of some in-pixel circuitry, a digitized photo-multiplier can be built in which every single-photon detection event is summed up by a counter. Moreover, this concurrent processing circuitry can be employed to realize low level image processing tasks. They can be efficiently implemented by this architecture given their intrinsic parallelism. Our proposal is to operate onto the light-induced signal at the focal plane in order to obtain a more elaborated record of the detection. For instance, by providing some characterization of the light spot. Information about the depth-of-interaction, in scintillation detectors, can be derived from the position and shape of the scintillation light distribution. This will ultimately have an impact on the spatial resolution that can be achieved. We are presenting the design in CMOS of an array of detector cells. Each cell contains a SPAD, an MOS-based passive quenching circuit and drivers for the column and row detection lines.
\end{abstract}

Keywords: SPAD, CMOS, focal-plane processing, depth of interaction, PET

\section{INTRODUCTION}

Functional imaging is a powerful diagnosis tool based on nuclear medicine techniques. Tracer molecules, labelled with radioactive isotopes, are introduced into the body and, according to the specific functionality of these molecules, they accumulate in the region/tissue/organ of interest. The radionuclides decay, emitting positrons that annihilate with atomic electrons. This is the principle behind positron emission tomography (PET). ${ }^{1}$ The annihilation of the positron with an electron leads to the emission of two high energy photons (gammarays) that depart in opposite directions. By examining the coincidences between detection events taking place at diametrically opposed sensors, visual information about the distribution of the tracer molecules can be extracted. The basic detector module employed in PET consists in a scintillator crystal -that converts the high energy photons into visible light photons- followed by a photo-multiplying device, ${ }^{2}$ as the scintillator signal is certainly faint. Typical timing and spatial resolutions required in PET are in the order of a few nanoseconds and below $4 \times 4 \mathrm{~mm}^{2}$. The most widely employed device for light amplification in PET detectors has been the photo-multiplier tube (PMT). Although the need for a higher spatial resolution and the operation within high magnetic fields has open the door for solid-state photodetectors. For instance, the single-photon avalanche diode (SPAD), ${ }^{3}$ also called avalanche diode in Geiger-mode. A SPAD detector consists in a diode biased above the breakdown voltage in which the incidence of a single photon triggers the avalanche current. Because this is a sustained phenomenon, the means for quenching the avalanche need to be provided. ${ }^{4}$ Interestingly, SPADs can be built in arrays, ${ }^{5}$ what allows for improved spatial resolution; and they are compatible with standard CMOS technology ${ }^{6} .{ }^{7}$ This permits the incorporation of processing circuitry in the same substrat following the smart imager concept, ${ }^{8}$ but this time applied to arrays of SPADs.

Further author information:

Francisco Pozas-Flores: IMSE-CNM-CSIC, C/ Americo Vespucio s/n 41092 Seville (Spain), Tel.: +34 954466666, Fax: +34 954466600, E-mail: pozas@imse-cnm.csic.es

\footnotetext{
Bioelectronics, Biomedical, and Bioinspired Systems V; and Nanotechnology V, edited by Ángel B. Rodríguez-Vázquez, Ricardo A. Carmona-Galán, Gustavo Liñán-Cembrano, Rainer Adelung, Carsten Ronning, Proc. of SPIE Vol. 8068, 806808 - (c) 2011 SPIE · CCC code: 0277-786X/11/\$18 - doi: 10.1117/12.888836
} 
As the avalanche diode biased above breakdown behaves as binary device, i. e. its possible states are either off or conducting a milliampere range current, it seems appropriate to treat its outputs as a time encoded signal. The basic cell, composed by the SPAD and the quenching and recharge circuitry, delivers voltage pulses representing photon detection events. These pulses can be summed up by a counter, along a prescribed time frame, constituting the so-called digital silicon photo-multiplier (SiPM).${ }^{9}$ By properly arranging the SiPM cells, information about the shape and dispersion of the light spot can be extracted concurrently with the detection process. This additional information will be useful for an accurate discrimination of coincident events, and therefore for achieving a higher spatial resolution. ${ }^{10}$

This paper is organized as follows. First we will review the different approaches to quenching that has been reported, and point out their advantages and drawbacks. Then we will introduce an hybrid approach in which the quenching device, a MOS transistor, operates as a resistor and a switch. After that we will explain the design of the basic cell of the array. Finally the overall operation of a small array generating profiles of photon incidence art the array clomuns and rows is illustrated with simulation results.

\section{PASSIVE AND ACTIVE QUENCHING}

A SPAD consists in a reverse biased p-n junction, whos bias voltage exceeds the breakdown voltage. In this state there is a bifurcation between two operating modes, the off branch of the I-V characteristic and the current avalanche branch. ${ }^{5}$ The incidence of a single photon in the depletion zone and the subsequent generation of an $e^{-} / h^{+}$pair is enough to trigger the current avalanche and send the device to the other branch of the characteristic. The avalanche current is a self-sustained phenomenon that can destroy the device if not quenched. For that purpose, a quenching circuit needs to be provided in order to decrease the voltage drop between the diode terminals, bringing it back to non-conduction. Once the avalanche current is stopped, another device -or the same- recharges the junction capacitance to the point in which a new incidence of a photon can be sensed. ${ }^{11}$

In order to design a SPAD with its quenching and recharging circuit, several features need to be taken into account, i. e. the avalanche probability, teh photon detection probability, the dynamic range, the probability of false counts, the jitter. These features depend on different parameters: the energy of the photons, the donor concentration, the bias voltage excess, the depth of the junction, the cleaness of the fabrication process. ${ }^{12}$ Most of these parameters are imposed by the characteristics of the process, not offering many possibilities to the designer to modulate the operation of the device. For instance, a small SPAD renders a low after-pulsing probability, lower jitter, shorter dead times and improved spatial resolution. In exchange, photon detection probability is low. Another user-controllable parameter is the excess voltage, i. e. the voltage above breakdown which biases the diode. If it is high, the detection probability increases as well as the timing resolution. However, there will be an increase in dark counts and after-pulses too. Therefore, device area and excess voltage will be the result of trading off some of the SPAD features. In addition to this, the mechanism and circuit selected for quenching also

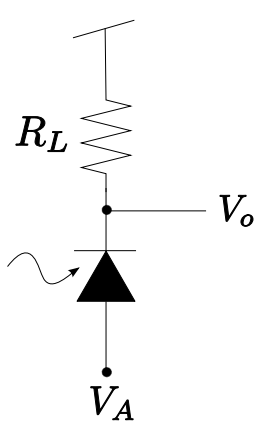

(a)

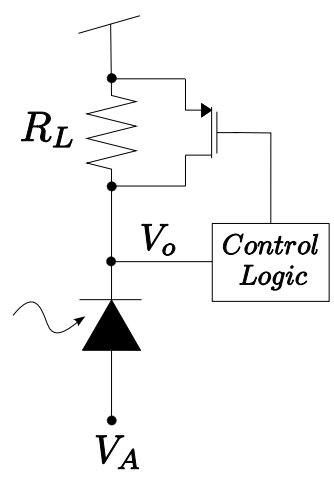

(b)

Figure 1: (a) Passive quenching and (b) active quenching circuits 
have an influence in the overall device performance. A slow quenching circuit limits the maximum counting rate while a large quenching circuit compromises the spatial resolution. The most simple way to stop the avalanche current in the diode is by using a high-value resistor connected in series (Fig. 1(a)), what is termed passive quenching. ${ }^{13}$ While the SPAD is in the off-branch, the voltage at $V_{o}$ is close to the power rail. When a photon is detected, so the SPAD enters in avalanche, the current flowing through $R_{L}$ causes a voltage drop across the resistor that makes the voltage $V_{o}-V_{A}$ fall below the breakdown voltage, thus interrupting the avalanche current. However, it is not $R_{L}$ what determines the duration of the quenching phase. During the avalanche, the diode can be modelled as a resistor, $R_{S P A D}$, in series with a voltage source, $V_{S P A D}$ (Fig. 3). Once the avalanche has started, the voltage between the anode and the cathode evolves following:

$$
C_{A C} \frac{d V_{A C}}{d t}=\frac{V_{d d}-V_{A C}}{R_{L}}-\frac{V_{A C}-V_{S P A D}}{R_{S P A D}}
$$

As the $R_{L}$ is much larger than $R_{S P A D}$, the first current contribution can be neglected and is the second one which determines the pace of the avalanche quenching. Once the diode is off again, node $V_{o}$ is recharged through $R_{L}$, recovering the power rail voltage, what biases the diode back above breakdown, letting it be able to detect a new photon impact. The value of $R_{L}$ needs to be high compared with the diode internal resistance in order to give rise to a voltage drop as large as the excess voltage. However, the larger $R_{L}$ the slower the recharging time. Being the diode in the off-branch:

$$
C_{A C} \frac{d V_{A C}}{d t}=\frac{V_{d d}-V_{A C}}{R_{L}}
$$

whose times constant, $R_{L} C_{A C}$ is large. In order to accelerate the recharging phase, it is necessary to introduce a different element that provides a less resistive path. One way to achieve this is to employ a MOS switch in parallel with the resistor (Fig. 1(b)). In this case, quenching is passive but recharging is active. ${ }^{12}$ The swicth is enabled by the output pulse with the appropriate logic. When the avalanche starts, $V_{o}$ drops from the power rail voltage down to the designed threshold. This event can be employed to trigger the activation of the recharge switch that allows for a faster recovery of the original voltage level at $V_{o}$. This configuration is known as active quenching. The dead-time of the SPAD, i. e. the period of time in which it is not able to detect any new incoming photon is:

$$
t_{\text {dead }}=t_{Q}+t_{D}+t_{R}
$$

what is the sum of the quenching and the recharging duration, $t_{Q}$ and $t_{R}$, plus the delay introduced by the recharging circuit logic, $t_{D}$. This last term is usually included in the recharge phase time.

Finally, it is also possible to provide active quenching using another switch. Reducing the quenching time decreases the probability of being affected by afterpulses. However, if the latency of the logic involved in the detection of the avalanche is large, the device is in fact passively quenched by the transistor operating as a resistor.

\section{MOS-BASED PASSIVE QUENCHING}

In order to reduce the area occupation of the quenching device, we are substituting the resistor $R_{L}$ by a $p$-type MOS transistor ( $M_{Q}$ in Fig. 2) operating in the ohmic region. Fine-tuning of voltage $V_{\text {bias }}$ will have an incidence of the detection probability, as it regulates how far beyond breakdown is the diode biased. Also it will influence the duration of the quenching phase, as it controls the build-up of the avalanche. In the mean-time, transistor $M_{R}$, that acts as a switch, remains off until the pulse in $V_{o}$ is detected. When the circuit is relaxed, $V_{o}$ approximates the power supply rail and the switch $M_{R}$ is off. Once the voltage pulse in $V_{o}$ is triggered, $M_{R}$ will rapidly recharge the node $V_{o}$ as it is much more conductive than $M_{Q}$. In order to verify this behavior by simulation we have employed a simplified version of a model reported in the literature ${ }^{13}$ (Fig. 3). We have modified the model parameters in order to account for the properties of a p-substrate/n-well diode built in a $0.18 \mu \mathrm{m}$ CMOS process. As displayed in the figure, the voltage source (modelled by $f(\cdot)$ ) and the SPAD resistance $\left(g_{2}(\cdot)\right)$ that model the avalanche-branch behavior are controlled by the voltage drop between the diode's cathode and anode (nodes $C$ and $A$ in Fig. 3, respectively). They are defined piecewise using data from the I-V characteristic of the diodes of the technology. It is necessary to emphasize here that we are presenting simulation results based on parameters extracted from the technology vendor characterization of the primitives they offer, which do not include a device 

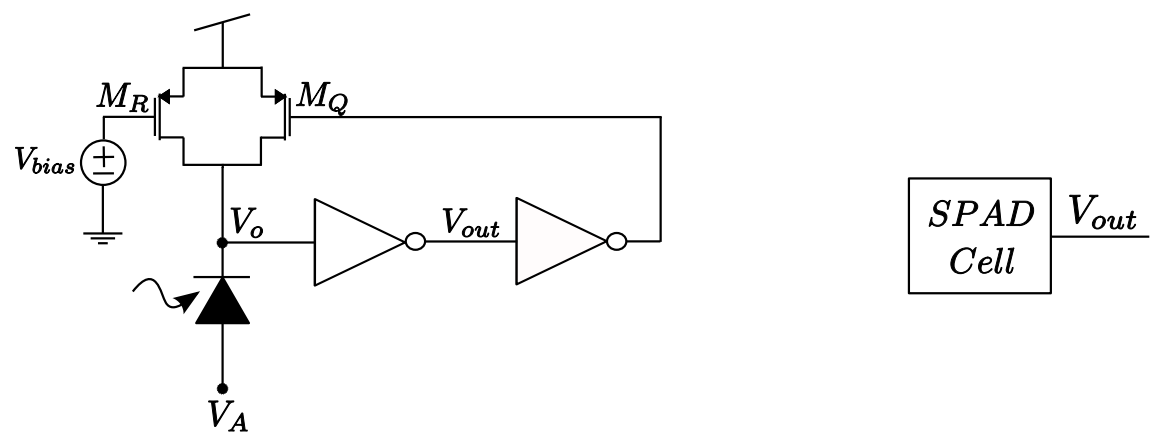

Figure 2: Quenching circuit based on a MOS-resistor and a single switch

like the SPAD. Therefore, we do not have experimental measurements on the devices yet. Having said this, the predicted advantages of the proposed quenching circuit are the elimination of the high-value resistor, and, like the rest of the circuits employing active recharging, a fast recharge phase.

Fig. 4 displays the response of the SPAD to a pulse representing the incidence of a photon in the depletion layer. The quenching transistor and the diode bias $\left(V_{A}\right)$ have been designed to render a voltage excursion at $V_{o}$ between the power supply rail $(3.3 \mathrm{~V})$ and the ground $(0 \mathrm{~V})$. These imput levels do not compromise the integrity of the detection logic. As shown $V_{o} u t$ increases, as $V_{o}$ decreases, to recover only after recharging. The diode current has a sudden increase, triggered by the photon pulse in the model, but is rapidly quenched as node $V_{o}$ is discharged. When comparing the simulated performace of the proposed circuit with already reported passive and active quenching circuits (Table 1), we have obtained dead times under the nanosecond. In order to make a fair comparison, the works reported ${ }^{1415}$ introduce hold-off circuitry to avoid afterpulsing. This is a phenomenon we have not contemplated in our simulations.

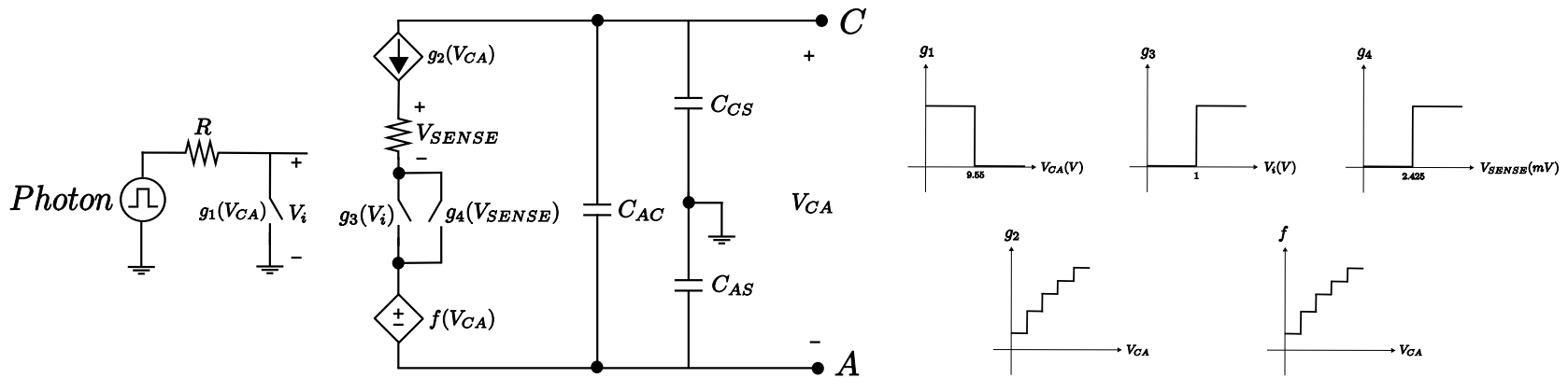

Figure 3: SPAD model

\begin{tabular}{|l|c|c|c|c|}
\hline Circuit features & Finkelstein $^{7}$ & Mita $^{14}$ & Grönholm $^{15}$ & This work \\
\hline Technology $(\mu \mathrm{m}) /$ Vdd $(\mathrm{V})$ & $0.18 / 3.3$ & $2.0 / 5.0$ & $0.13 / 2.5$ & $0.18 / 3.3$ \\
\hline Area $\left(\mu \mathrm{m}^{2}\right)$ & 38.48 (ext. res.) & 5000 & - & 38.44 \\
\hline Dead time & $2 \mathrm{~ns}$ & $4 \mathrm{~ns}$ & $7.5 \mathrm{~ns}$ & $667 \mathrm{ps}$ \\
\hline Quenchig type & passive & active & active & passive (MOS) \\
\hline Quenchig time & - & $3 \mathrm{~ns}$ & - & $84 \mathrm{ps}$ \\
\hline Recharging time & - & $1 \mathrm{~ns}$ & - & $583 \mathrm{ps}$ \\
\hline Peak diode current & $3 \mathrm{~mA}$ & - & - & $3.73 \mathrm{~mA}$ \\
\hline Bias/breackdown voltages $(\mathrm{V})$ & $17 / 10$ & $20 /-$ & $-/-$ & $12.95 / 9.55$ \\
\hline
\end{tabular}

Table 1: Comparison between quenching/recharge circuits 


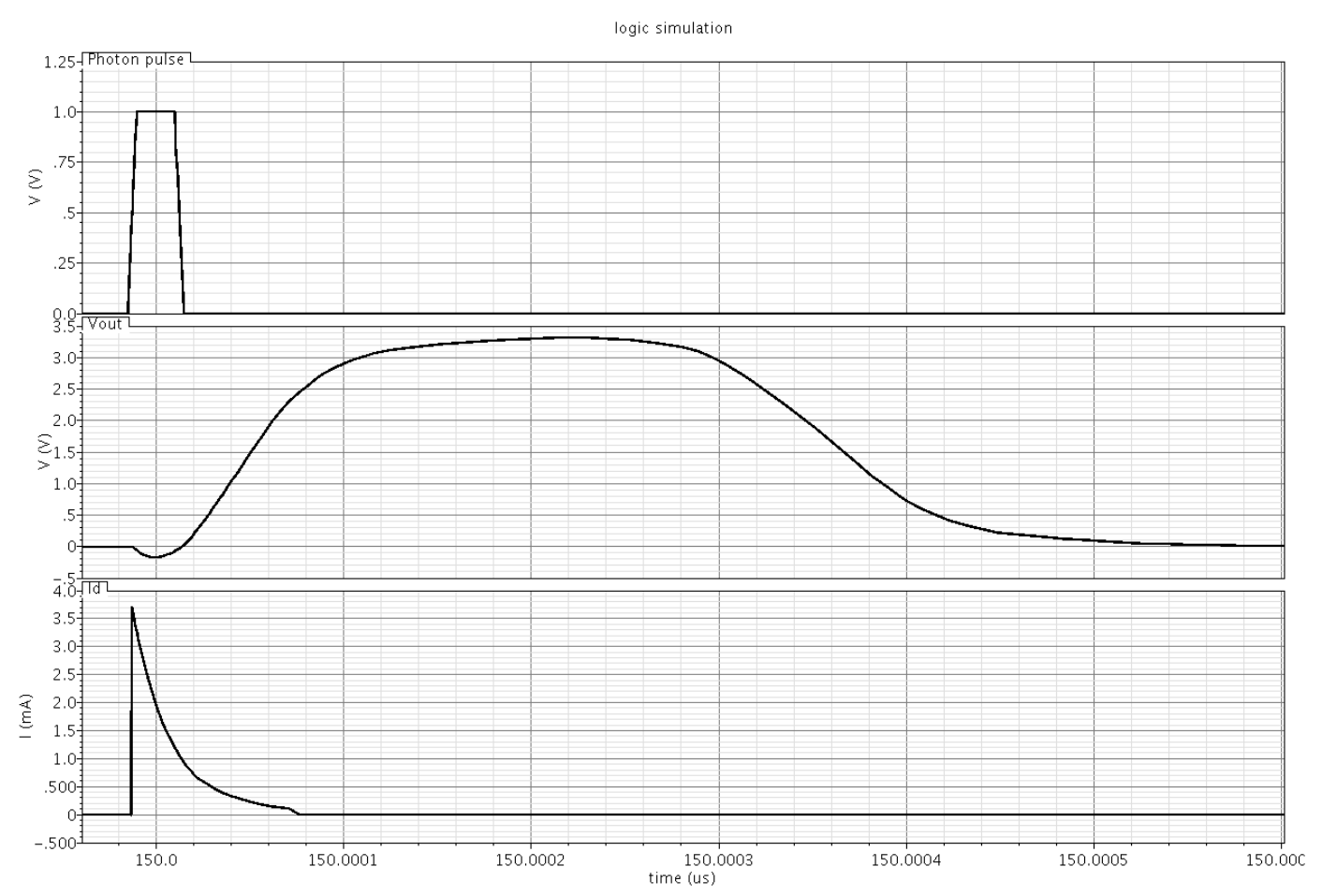

Figure 4: Waveforms of the response of the SPAD: voltage $V_{o} u t$ and diode current

\section{ON-CHIP FEATURE EXTRACTION}

SPAD arrays are employed in PET for detecting visible light photons at the scintillation crystals, as a product of the energy transfer from incident $\gamma$-rays. ${ }^{16}$ SPADs arrays are placed at either one or the two ends of the crystal. $^{2}$ The complete array can operate as a single-device, therefore adding up the current contribution of all the devices; ${ }^{17}$ or they can operate as position-sensitive detectors, ${ }^{18}$ at an improved spatial resolution. In real detectors, high energy photons suffer various scatterings within the scintillator before being finally absorbed. This leads to the generation of several photons. This fact and the repeated incidence of gamma-rays coming from a specific point in the sample, allows to think in a distribution of photon impacts in the surface of the SPAD arrays. The particular shape and charcateristics of this light-distribution is connected with the depth of interaction (DOI) within the crystal, ${ }^{10}$ what is of importance in order to establish an accurate line-of-response (LOR). The more precise the determination of the LOR the better image quality/spatial resolution.

Suppose that an array of cells like that in Fig. 2 is built following the scheme in Fig. 5 . The output voltage of each cell, $V_{\text {out }}$, is employed to drive a couple of transistors that pull down the voltage at the row and column lines. These voltages are tied to $V_{d d}$ with the help of a pull-up transistor. Each time that any of the SPADs in the row or column detects a photon, it generates a positive pulse that drives down the row or column voltage. This change is registered by a counter. The rows and columns of the array act as independent digital silicon photo-multipliers. The counters need to be sensitive and fast enough to account for every detection. After the appropriate observation period, the number of hits at every row/column of the array constitute an histogram -Radon transform- of the activity in that row/column. Statistics on these histograms can be employed to determine the center of mass and the dispersion of the light-distribution. Consider a square array of $N \times N$ pixels. Let us assume that $i \in\{1, \ldots, N\}$ is the position of the histogram bin, i. e. the number of the row or column of the array. Also $p_{i}$ is the probability of that row or column of detectors of being hit. In other words, the number of hits in that specific row or column divided by the total number of hits. Then:

$$
\mu_{1}=\sum_{i=1}^{N} i p_{i}
$$




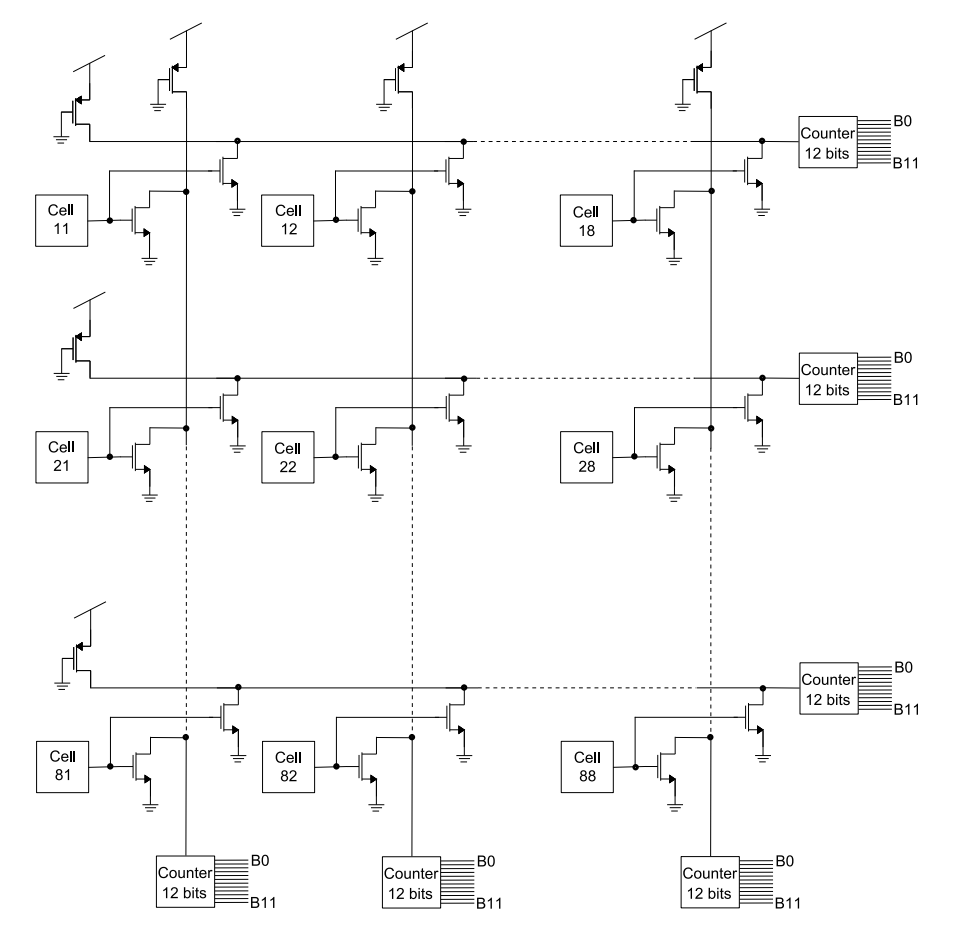

Figure 5: Array of SPADs in the form of row and column digital SiPMs

is the center of mass of the 1-D distribution. While:

$$
\mu_{2}=\sum_{i=1}^{N}\left(\mu_{1}-i\right)^{2} p_{i}
$$

is the variance or second central moment. Assuming that the scintillation photons are emitted isotropically from a point-source and that the fraction of this produced light that is reabsorbed by the scintillation crystal is negligible, it can be determined ${ }^{10}$ that $\sigma$, the standard deviation of the light-distribution, is directly related with the DOI through a power law of the form:

$$
z-z_{0} \propto\left(\sigma-\sigma_{0}\right)^{2}
$$

Fig. 6 depicts the simulated on-line characterization of the light-spot by a $8 \times 8$-pixel array. The grayscale image illustrate the amount of hits registered at each row or column, while the waveforms correspond to the row and column voltages.

\section{CONCLUSIONS}

The design of an array of SPADs for improved spatial resolution PET imaging is presented. It is realized in $0.18 \mu \mathrm{m}$ standard CMOS. Rows and columns of the array behave as independent digital silicon photo-multipliers. The outcome of the array processing are the histograms of the rows and columns of teh array. This information is useful to compute the DOI and therefore to achieve a clearer picture of the sample.

\section{ACKNOWLEDGMENTS}

This work is partially funded by the Andalusian Regional Government through project 2006-TIC-2352, by the Spanish Ministry of Science and Innovation through project TEC 2009-11812, co-funded by the European Regional Development Fund, and also supported by the Office of Naval Research (USA), through grant N000141110312. 


\section{REFERENCES}

[1] Iniewski, K., [Medical imaging: principles, detectors, and electronics], Wiley (2009).

[2] Lewellen, T., "Recent developments in PET detector technology," Physics in medicine and biology 53, R287 (2008).

[3] Aull, B. F., Loomis, A. H., Young, D. J., Heinrichs, R. M., Felton, B. J., Daniels, P. J., and Landers, D. J., "Geiger-mode avalanche photodiodes for three-dimensional imaging," Lincoln Laboratory Journal 13(2), 335-350 (2002).

[4] Zappa, F., Ghioni, M., Cova, S., Samori, C., and Giudice, A., "An integrated active-quenching circuit for single-photon avalanche diodes," IEEE Transactions on Instrumentation and Measurement 49(6), 1167 -1175 (2000).

[5] Zappa, F., Tisa, S., Tosi, A., and Cova, S., "Principles and features of single-photon avalanche diode arrays," Sensors and Actuators A: Physical 140(1), 103-112 (2007).

[6] Niclass, C., Sergio, M., and Charbon, E., "A single photon avalanche diode array fabricated in deepsubmicron CMOS technology," in [Proceedings of the Design, Automation and Test in Europe Conference], 1, 1-6 (2006).

[7] Finkelstein, H., Hsu, M., and Esener, S., "STI-bounded single-photon avalanche diode in a deepsubmicrometer cmos technology," IEEE Electron Device Letters 27(11), 887 -889 (2006).

[8] Ohta, J., [Smart CMOS image sensors and applications], CRC Press (2007).

[9] Frach, T., Prescher, G., Degenhardt, C., de Gruyter, R., Schmitz, A., and Ballizany, R., "The digital silicon photomultiplier - principle of operation and intrinsic detector performance," in [IEEE Nuclear Science Symposium], 1959 -1965 (2009).

[10] Lerche, C., Benlloch, J., Sanchez, F., Pavon, N., Escat, B., Gimenez, E., Fernandez, M., Torres, I., Gimenez, M., Sebastia, A., and Martinez, J., "Depth of gamma;-ray interaction within continuous crystals from the width of its scintillation light-distribution," IEEE Transcations on Nuclear Science 52(3), 560 - 572 (2005).

[11] Tisa, S., Zappa, F., Tosi, A., and Cova, S., "Electronics for single photon avalanche diode arrays," Sensors and Actuators A: Physical 140(1), 113-122 (2007).

[12] Rochas, A., Single Photon Avalanche diodes in CMOS technology, PhD thesis, EPFL (2003).

[13] Zappa, F., Tosi, A., Mora, A., and Tisa, S., "SPICE modeling of single photon avalanche diodes," Sensors and Actuators A: Physical 153(2), 197-204 (2009).

[14] Mita, R. and Palumbo, G., "High-speed and compact quenching circuit for single-photon avalanche diodes," IEEE Transactions on Instrumentation and Measurement 57(3), 543 -547 (2008).

[15] Grönholm, M., Poikonen, J., and Laiho, M., "A ring-oscillator-based active quenching and active recharge circuit for single photon avalanche diodes," in [European Conference on Circuit Theory and Design], 5 -8 (2009).

[16] Fishburn, M. and Charbon, E., "System tradeoffs in gamma-ray detection utilizing SPAD arrays and scintillators," IEE Transactions on Nuclear Science 57(5), 2549 -2557 (2010).

[17] Llosa, G., Lacasta, C., Belcari, N., Bisogni, M., Collazuol, G., Marcatili, S., Barrillon, P., Bondil-Blin, S., de la Taille, C., Piemonte, C., and Del Guerra, A., "Monolithic 64-channel SiPM matrices for small animal PET," in [IEEE Nuclear Science Symposium], 2658 -2661 (2009).

[18] Dokhale, P. A., Silverman, R. W., Shah, K. S., Grazioso, R., Farrell, R., Glodo, J., McClish, M. A., Entine, G., Tran, V.-H., and Cherry, S. R., "Performance measurements of a depth-encoding PET detector module based on position-sensitive avalanche photodiode read-out," Physics in Medicine and Biology 49(18), 4293 (2004). 

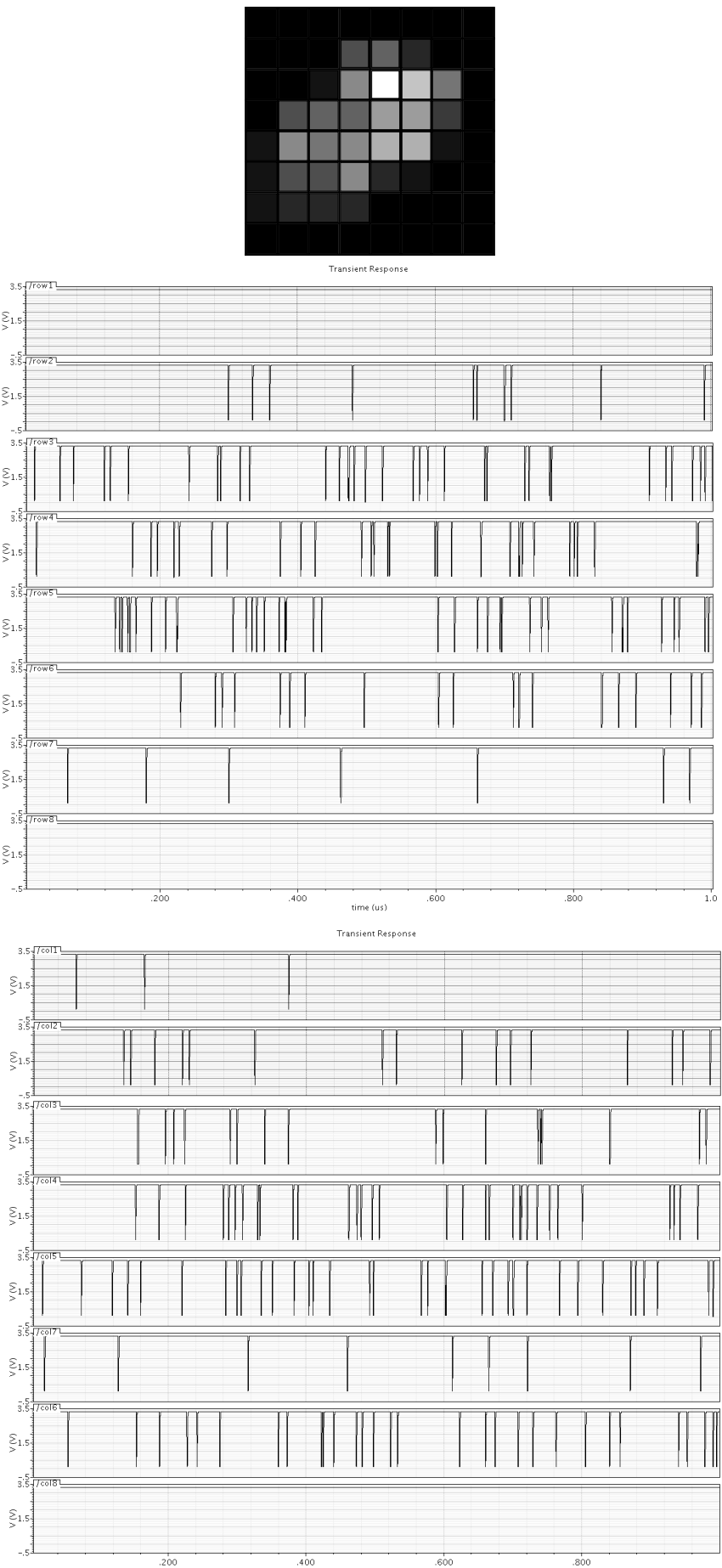

Figure 6: Waveforms of the row and column lines of the SPAD array

Proc. of SPIE Vol. 8068 806808-8 